\title{
REFERENCE CROSS SECTIONS RELEVANT FOR NEUTRON DETECTORS
}

\author{
J. Blomgren* \\ Department of Neutron Research \\ Uppsala University \\ Box 525, S-751 20 Uppsala, Sweden \\ E-mail: Jan.Blomgren@tsl.uu.se
}

\begin{abstract}
Neutron detection inevitably proceeds via nuclear reactions, making nuclear cross section data of major importance in the determination of neutron detector properties in general, and efficiency determination in particular. Up to about $20 \mathrm{MeV}$, the cross section data base is fairly advanced, and the codes for, e.g., determination of scintillator properties produce results with uncertainties on the level of a few percent. Above $20 \mathrm{MeV}$, the scarcity of nuclear data results in much larger uncertanties in model calculations of detector performances.

The data base above $20 \mathrm{MeV}$ is meagre, but this is not the only problem. It also contains severe discrepancies for the most important cross sections. In this energy domain, the neutron-proton $(n p)$ scattering cross section is used as primary standard. The $n p$ scattering database contains discrepancies up to about $10 \%$. Since essentially all other cross sections are measured relative to $n p$ scattering, this means that most data have similar uncertainties. In the talk, the present status of reference cross sections in general and $n p$ scattering in particular is outlined, and recent experiments to elucidate the problems are presented. Moreover, the data situation concerning fast-neutron fission as reference is discussed.
\end{abstract}

International Workshop on Fast Neutron Detectors and Applications

April, 3 - 6, 2006

University of Cape Town, South Africa

${ }^{*}$ Speaker. 


\section{Introduction}

To measure a cross section, the intensity of the beam has to be known. For charged beams, this is a straight-forward affair, but to determine a neutron beam intensity is actually very difficult.

A charged particle interacts with the electrons of the atom. Thereby it is possible to build systems where every particle gives a signal when passing through a detector, and hence it is a relatively simple task to determine the beam intensity by just counting pulses. Another option is to stop particles via their energy loss - which is also an effect of interaction with the atomic electrons - and finally measure the collected charge.

Neutrons interact by the strong interaction only, and they are uncharged. This means that there is no way you can build a device which produces a signal for each particle that passes, and you cannot stop neutrons in a controlled way. Detection of neutrons always has to proceed via a nuclear reaction, releasing charged particles, which can subsequently be detected. The problem is that there is no way to determine a nuclear cross section from theory only with a reasonable precision. This means we end up in circular reasoning.

Let us assume we want to use neutron-proton $(n p)$ scattering for neutron detection. Counting the protons emanating from a hydrogenous material is a simple task, but we need to know the cross section to derive the number of incoming neutrons. To measure that cross section, however, we need to know the number of incident neutrons. Are there no ways out of this vicious circle?

In fact, there are a few tricks which can be used, but they are all associated with large difficulties. The standard procedure is therefore to determine a single cross section using all these painstaking methods, and subsequently this cross section is used as reference, i.e., other cross section measurements are measured relative to it. The only three techniques available are presented below:

1. Tagged beams. The methodologically simplest method is probably to use tagged beams, but this does not necessarily mean it is the simplest technique in real life. For a few reactions, detection of the residual nucleus can be used to verify the neutron production. An example is the $\mathrm{D}(\mathrm{d}, \mathrm{n})^{3} \mathrm{He}$ reaction. By detecting the kinetic energy and direction of the residual ${ }^{3} \mathrm{He}$ nucleus, the energy and angle of the neutron is known. In addition, the detection of a ${ }^{3} \mathrm{He}$ nucleus implies that there must be a neutron, i.e., the ${ }^{3} \mathrm{He}$ nucleus serves as a "tag" on the neutron. With this technique, "beams" of really low - but well-known - intensity can be produced. This beam can subsequently be used for cross section measurements.

2. Combination of total and differential hydrogen cross sections. The total cross section, i.e., the probability that a neutron interacts at all with a target nucleus, is a quantity that can be determined without knowledge of the absolute beam intensity. This integral cross section is related to the attenuation of a neutron beam, which means that a relative measurement of the beam intensity before and after a target is sufficient.

In the case of hydrogen, the total cross section is completely dominated by elastic $n p$ scattering, which accounts for more than $99 \%$ of the total cross section. A relative measurement of the $n p$ scattering angular distribution can thereby be normalized to agree with the total $n p$ cross section, and thus an absolute $n p$ differential cross section can be obtained. 
3. Combination of total, reaction and differential elastic cross sections. The differential elastic cross section of a nucleus can be determined absolutely by a combination of total and reaction cross section measurements, together with a relative measurement of the differential elastic cross section. Both the total cross section and the reaction cross section can be determined in relative measurements of beam attenuation. The only important difference is the geometry used. The integrated elastic cross section can then be derived as the difference of the total and reaction cross sections. The elastic differential cross section on almost any nucleus falls dramatically with angle. Thus, by covering a moderately wide range at forward angles, essentially all the elastic differential cross section is covered. Thereby, the differential cross section can be related to the integrated elastic cross section.

The primary standard reference cross section for fast neutrons is $n p$ scattering. Instead of trying to measure a certain cross section on an absolute scale, a typical experiment would be designed to measure the ratio of that cross section versus $n p$ scattering. This facilitates the experimental work immensely.

Although proton recoil detection from $n p$ scattering is the most commonly used normalization technique, not all reactions are well suited to be measured against $n p$ scattering. Therefore, secondary standards have been proposed. This is particularily true for fission studies, for which ${ }^{209} \mathrm{Bi}$, ${ }^{235} \mathrm{U}$ and ${ }^{238} \mathrm{U}$ have been used. Fission is very useful for beam intensity monitoring, because the products to detect, the fission fragments, can hardly be created due to any type of background, and they are easily separated from other types of products created in the target, like $\alpha$ particles.

A major risk with relative measurements is that if the reference cross section is incorrect, all other data measured relative to it will be equally off. It turns out that for neutrons above $50 \mathrm{MeV}$, there have been quite some problems with both the primary and the secondary standards lately.

\section{Neutron-proton scattering}

The $n p$ scattering cross section - in particular at $180^{\circ}$ (c.m.), which corresponds to proton emission at $0^{\circ}$ in the lab - is frequently used to normalize measurements of other neutron-induced cross sections, i.e., it is the primary standard cross section. In addition, it plays an important role in fundamental physics, because it can be used to derive a value of the absolute strength of the strong interaction in the nuclear sector, commonly expressed as the pion-nucleon coupling constant, $g_{\pi N N}^{2}$ (see Ref. [1] for a review). Large uncertainties for such an important cross section are therefore unacceptable.

Unfortunately, there are severe discrepancies in the data base on $n p$ scattering in the 100-1000 $\mathrm{MeV}$ range [2], and uncertainties of $10 \%$ or more are common. These discrepancies concern both the shape of the angular distributions and the absolute normalization, and in fact there is no combination of two major experiments that agree. The experimental data discussed in the present paper are representative to the situation, but there are many more data sets available.

The $n p$ scattering data base was until recently dominated by the Bonner et al. data $(160-800$ $\mathrm{MeV}$ ) from Los Alamos [3] and essentially all evaluations and theory work in the field were fitted to those results. Recently, the results of two high-precision experiments, at TSL Uppsala at 96 $\mathrm{MeV}$ [4] and $162 \mathrm{MeV}$ [5], and at PSI [6] (200 - $580 \mathrm{MeV})$, have been published. The Uppsala 

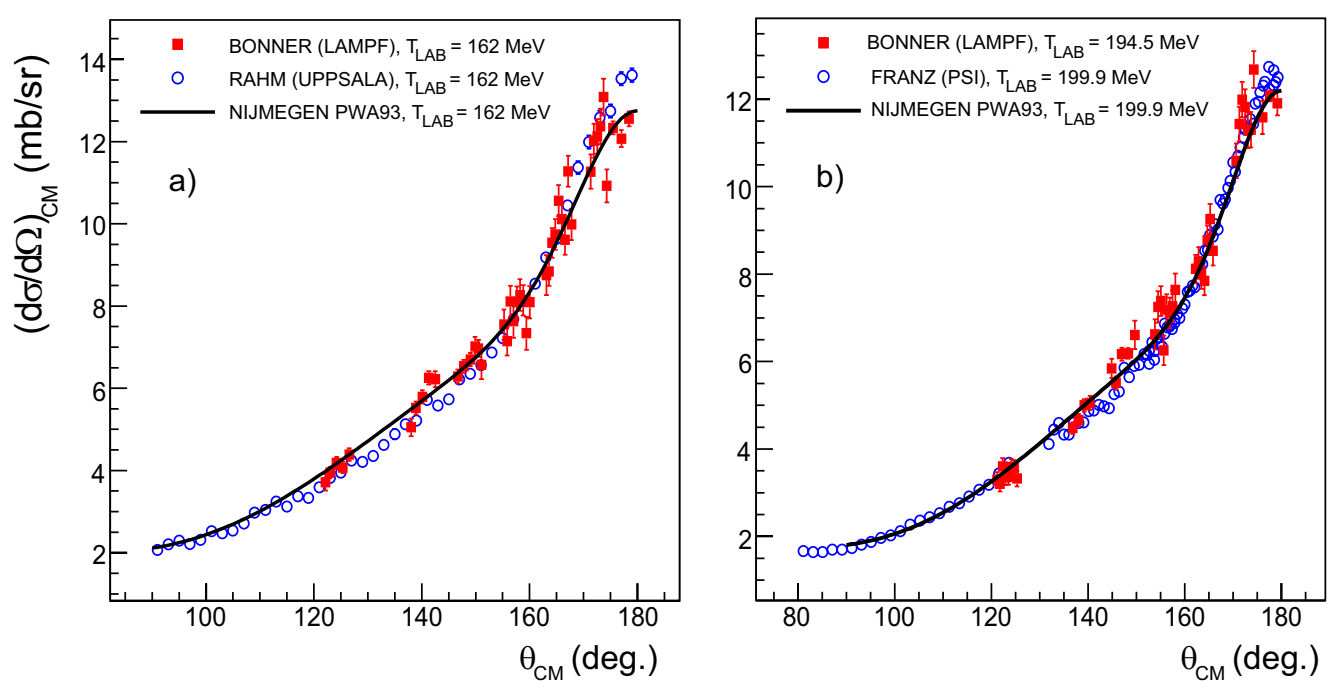

Figure 1: Comparison of $n p$ scattering differential cross section measurements (left panel) from Uppsala [4] and Los Alamos [3], and (right) from PSI [6] and Los Alamos near $200 \mathrm{MeV}$. The Los Alamos data are in each case represented by closed squares and the other data by open circles. The experimental results are compared to the Nijmegen PWA93 [9] partial wave analysis solution. The Los Alamos data have been renormalized by factors of 1.092 (left) and 1.078 (right) to bring them into agreement with the PWA. The PSI cross sections have been similarly normalized here, while the reported absolute cross section scale for the Uppsala data has been retained.

and PSI experiments agree in shape (which is a rather weak function of beam energy), but not in absolute magnitude, and both show a steeper angular distribution at backward angles than the Bonner data. The different absolute scale can, however, be attributed to different normalization methods. If the same normalization procedure is applied to the two sets, they are in agreement. Thus, there is a problem of inconsistencies in magnitude as well as in shape at the most backward angles (see fig. 1).

All the experiments above were performed using magnetic spectrometers for proton recoil detection and a neutron beam of unknown intensity, i.e., all data sets have been normalized after the experiment. The Uppsala data were normalized to the total cross section, while the other two experiments were normalized to a pion-production reaction. The latter method can only be used above $275 \mathrm{MeV}$ (the pion production threshold) and it depends on several corrections of unknown precision.

Recently, the entire problem has been addressed at IUCF with a novel technique [7, 8]. Tagged neutrons have been produced at $194 \mathrm{MeV}$ using the $\mathrm{D}(\mathrm{p}, \mathrm{n})^{2} \mathrm{He}$ reaction, where ${ }^{2} \mathrm{He}$ denotes two protons in a ${ }^{1} S_{0}$ state, i.e., by detecting two correlated low-energy protons, the energy and direction of the outgoing neutron was identified event-by-event. Proton recoils from these tagged neutrons impinging on a scintillator target were detected in a large scintillator array equipped with wire chamber tracking.

The results are shown in fig. 2. The new tagged IUCF data ("present experiment" in the figure) agree well with a PWA fitted to the shape (but not magnitude) of the Bonner data. If believing 


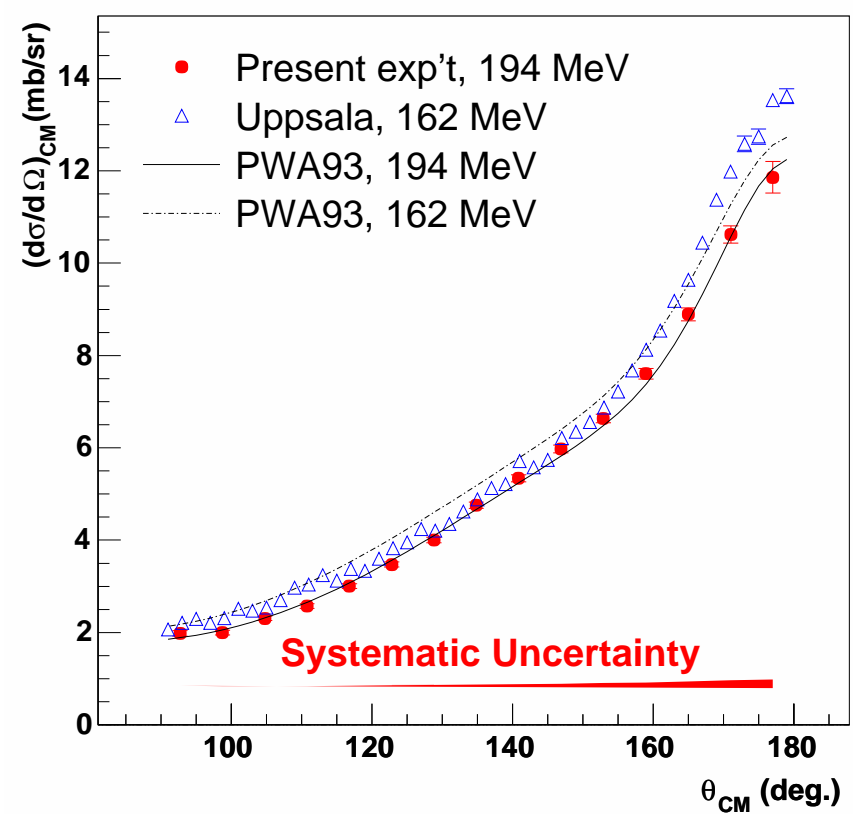

Figure 2: Absolute differential cross section from the IUCF experiment at $194 \mathrm{MeV} \mathrm{[7,} \mathrm{8],} \mathrm{compared} \mathrm{with}$ data from TSL Uppsala [5] and with PWA calculations [9] at two relevant energies. The figure is taken from ref. [8], i.e., "present experiment" refers to ref. [8]. The error bars on the present results are statistical (including background subtraction), while the shaded band represents all systematic uncertainties, including those in the overall normalization.

the new tagged data (and my personal opinion is that they are probably the best data available), it can be conluded that the normalization technique used by the TSL Uppsala group is in good agreement with the new IUCF data. As mentioned previously, the Los Alamos and PSI data need significant renormalization to agree with the PWA that describes the IUCF data well (see fig. 1). Thus, normalization to the total $n p$ cross section seems to be a better technique than using pion production as reference.

The shape of the angular distribution, however, is in better agreement with the Bonner data than the Uppsala and PSI experiments. To clear up this controversy once for all, it would be valuable to find an explanation why two recent experiments (Uppsala and PSI) agree internally in shape with a steeper angular distribution, but only at the most backward angles. For almost all the angular range, all the experiments agree in shape. Moreover, producing a too steep angular distribution is not straight-forward; most experimental problems tend to reduce steepness. It is proposed in a forthcoming IUCF publication [8] that this could be due to either not taking the neutron beam divergence properly into account or to a mismatch of the proton recoil solid angles near zero degrees in the laboratory system. Investigations of this effect for the Uppsala data are underway. 


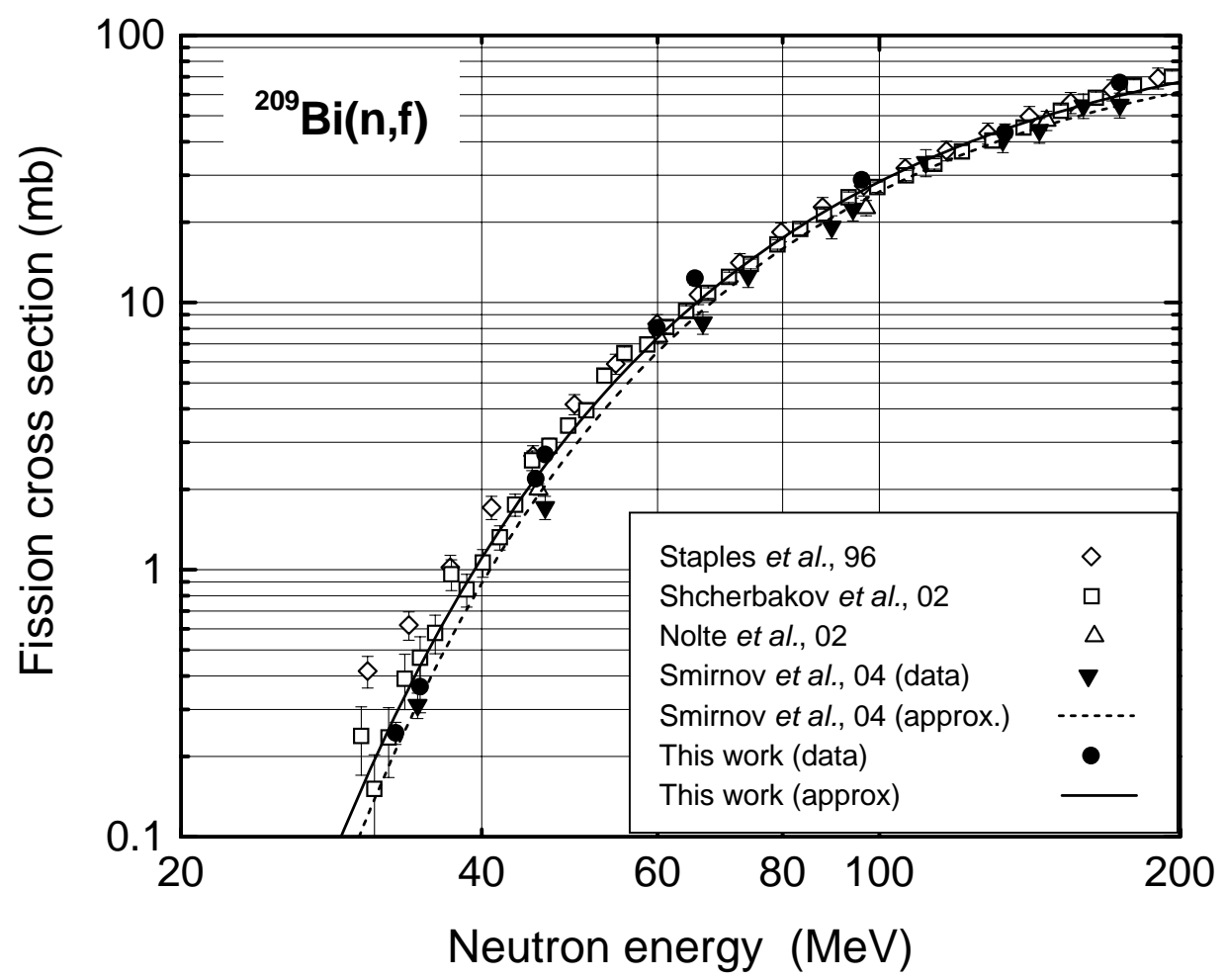

Figure 3: Measured neutron-induced fission cross sections of ${ }^{209} \mathrm{Bi}[13,14,15,16,17]$. The figure is taken from ref. [13], i.e., "present experiment" refers to ref. [13]. The data from Refs. [16, 17] are given with regard to the recommended standard uncertainties [10]. The solid and dashed lines show the cross section parameterizations of Ryzhov et al. [13] and of Smirnov et al. [14], resp.

\section{Fission}

When it comes to fission cross section data, not much is available above $20 \mathrm{MeV}$. As described above, fission chambers are frequently used for beam monitoring. As long as relative monitoring is the only ambition, precise knowledge of the cross section is not very important. General technical reliability is more important. One critical issue, however, is the presence of thermal neutrons. The thermal neutron content in a fast-neutron beam is often unknown, and so is the ambient thermal background. Moreover, this thermal background is often not stable, and therefore fissile nuclides are preferentially not used for fast-neutron monitoring. Thus, ${ }^{238} \mathrm{U}$ is more often used than ${ }^{235} \mathrm{U}$.

The readily available nuclides ${ }^{232} \mathrm{Th}$ and ${ }^{238} \mathrm{U}$ both have fission thresholds of about $1 \mathrm{MeV}$. The next lighter elements that are available for realistic applications are lead and bismuth, the heaviest stable elements, which have effective fission thresholds of about $20-30 \mathrm{MeV}$. In between bismuth and thorium, all elements have rather short halflives and are therefore difficult to handle and hard to obtain in useful quantities. This limits the practical possibilities to detectors with thresholds either in the 1 or $20 \mathrm{MeV}$ range. 
For applications of very fast neutrons, above $20 \mathrm{MeV}$, it is often of interest to have a high threshold to reduce the influence of neutrons below $20 \mathrm{MeV}$. For such applications, the ${ }^{209} \mathrm{Bi}(\mathrm{n}, \mathrm{f})$ cross section has been recommended as a secondary neutron cross section standard [10]. Bismuthbased neutron fluence monitors, being insensitive to low-energy $(<25 \mathrm{MeV})$ neutrons, are used in experiments where the low-energy neutron background is difficult to determine [11, 12].

Meanwhile, the present uncertainty of the ${ }^{209} \mathrm{Bi}(\mathrm{n}, \mathrm{f})$ standard is rather large (see fig. 3), so further improvement of the standard is necessary. In a recent paper, Ryzhov et al. [13] presents a parametrization to the existing data. A similar parametrization has previously been proposed by Smirnov et al. [14]. These two parameterization differ by about $10-15 \%$ in the $50-150 \mathrm{MeV}$ range. It is notable, however, that the shapes of the two resulting functions are in fair agreement. To some extent it can be a normalization effect. It should be noted that different normalization techniques have been used for the various experiments in the data base. The data of Ryzhov et al. [13] and of Smirnov et al. [14] were normalized to the ${ }^{238} \mathrm{U}(\mathrm{n}, \mathrm{f})$ cross section standard, while Nolte et al. [15] normalized to $n p$ scattering (except one point at $97 \mathrm{MeV}$ ) and Shcherbakov et al. [16] to the ${ }^{235} \mathrm{U}(\mathrm{n}, \mathrm{f})$ cross section.

It should be pointed out that the experiments normalized to ${ }^{235} \mathrm{U}$ and ${ }^{238} \mathrm{U}$ are implicitly normalized to something else, because these cross sections cannot be determined absolutely. Some of the high-energy fission data on uranium isotopes are normalized using $n p$ scattering as reference, but some data have used other methods, sometimes of unknown quality. I have chosen to use the data situation for lead and bismuth as an illustration, but the data quality is similar for uranium. Also there, $10 \%$ uncertainties are common.

Thus, work to establish accurate cross section standards is highly motivated. For instance, at TSL Uppsala a project is underway in which the ${ }^{238} \mathrm{U}(\mathrm{n}, \mathrm{f})$ cross section will be measured relative to $n p$ scattering using the same detectors and target (two layers, ${ }^{238} \mathrm{U}$ with a $\mathrm{CH}_{2}$ backing) for simultaneous detection of proton recoils and fission fragments. With such a technique, some of the systematic uncertainties of previous experiments cancel.

\section{Conclusions}

Both the primary and the secondary standards have large uncertainties. Up to now, it has been reasonable to ascribe 5-10\% uncertainty to the $n p$ scattering cross section and $10-15 \%$ to the best-known fission cross sections. There are hopes that the situation for $n p$ scattering can be improved upon. A recent experiment has shed new light on the problem. If follow-up investigations can elucidate the origin of previous discrepancies in the data base, it might be possible to arrive at a $3 \%$ uncertainty in $n p$ scattering. This could in turn allow fission cross sections to be determined to about $5 \%$ uncertainty. New high-precision experiments are most welcome to allow us to reach this goal.

\section{Acknowledgments}

This work was financially supported by the Swedish Nuclear Fuel and Waste Management Company, the Swedish Nuclear Power Inspectorate, Ringhals AB, Forsmarks Kraftgrupp AB, the Swedish Defense Research Agency, the Swedish Nuclear Safety and Training Centre, and the European Union. 


\section{References}

[1] Proceedings of Workshop on "Critical Points in the Determination of the Pion-Nucleon Coupling Constant", Uppsala, June 7-8, 1999, ed. J. Blomgren. Phys. Scr. T87 (2000).

[2] J. Blomgren, N. Olsson, J. Rahm, Phys. Scr. T87, 33 (2000).

[3] B.E. Bonner, J.E. Simmons, C.L. Hollas, C.R. Newsom, P.J. Riley, G. Glass, Mahavir Jain, Phys. Rev. Lett. 41, 1200 (1978).

[4] J. Rahm, J. Blomgren, H. Condé, S. Dangtip, K. Elmgren, N. Olsson, T. Rönnqvist, R. Zorro, O. Jonsson, L. Nilsson, P.-U. Renberg, A. Ringbom, G. Tibell, S.Y. van der Werf, T.E.O. Ericson, B. Loiseau, Phys. Rev. C63, 044001 (2001).

[5] J. Rahm, J. Blomgren, H. Condé, S. Dangtip, K. Elmgren, N. Olsson, T. Rönnqvist, R. Zorro, A. Ringbom, G. Tibell, O. Jonsson, L. Nilsson, P.-U. Renberg, T.E.O. Ericson, B. Loiseau, Phys. Rev. C57, 1077 (1998).

[6] J. Franz, E. Rössle, H. Schmitt, L. Schmitt, Phys. Scr. T87, 14 (2000).

[7] M. Sarsour, T. Peterson, M. Planinic, S.E. Vigdor, C. Allgower, B. Bergenwall, J. Blomgren, T. Hossbach, W.W. Jacobs, C. Johansson, J. Klug, A.V. Klyachko, P. Nadel-Turonski, L. Nilsson, N. Olsson, S. Pomp, J. Rapaport, T. Rinckel, E.J. Stephenson, U. Tippawan, S.W. Wissink, Y. Zhou, Phys. Rev. Lett. 94 (2005) 082303.

[8] M. Sarsour, T. Peterson, M. Planinic, S.E. Vigdor, C. Allgower, B. Bergenwall, J. Blomgren, T. Hossbach, W.W. Jacobs, C. Johansson, J. Klug, A.V. Klyachko, P. Nadel-Turonski, L. Nilsson, N. Olsson, S. Pomp, J. Rapaport, T. Rinckel, E.J. Stephenson, U. Tippawan, S.W. Wissink, Y. Zhou, Measurement of the Absolute Differential Cross Section for np Elastic Scattering at $194 \mathrm{MeV}$, submitted to Phys. Rev. C.

[9] V.G.J. Stoks, R.A.M. Klomp, M.C.M. Rentmeester, and J.J. de Swart, Phys. Rev. C 48, 792 (1993).

[10] A.D. Carlson, S. Chiba, F.-J. Hambsch, N. Olsson, and A.N. Smirnov, IAEA Report INDC (NDC)-368, Vienna, 1997; Proc. Int. Conf. on Nuclear Data for Science and Technology, Trieste, Italy, 1997, Part II, p.1223.

[11] W. Glasser, R. Michel, S. Neumann, H. Schuhmacher, V. Dangendorf, R. Nolte, U. Herpers, A.N. Smirnov, I.V. Ryzhov, A.V. Prokofiev, P. Malmborg, D. Kollár, J.P. Meulders, J. Nucl. Sci. Tech. Suppl. 2, vol. 1 (2002) 373.

[12] R. Michel, W. Glasser, U. Herpers, H. Schuhmacher, H.J. Brede, V. Dangendorf, R. Nolte, P. Malmborg, A.V. Prokofiev, A.N. Smirnov, I.V. Ryzhov, J.P. Meulders, M. Duijvestijn, and A. Koning, Proc. Int. Conf. on Nuclear Data for Science and Technology, Santa Fé, NM, USA, 2004, AIP Conf. Proc. 769 (2005) 861.

[13] I.V. Ryzhov, G.A. Tutin, A.G. Mitryukhin, S.M. Soloviev, J. Blomgren, P.-U. Renberg, J.-P. Meulders, Y. El Masri, Th. Keutgen, R. Preels, R. Nolte, Measurement of neutron-induced fission cross sections of ${ }^{205} \mathrm{Tl},{ }^{204,206,207,207} \mathrm{~Pb}$ and ${ }^{209} \mathrm{Bi}$ with a multi-section Frisch-gridded ionization chamber, accepted for publication in Nucl. Instr. Meth. A.

[14] A.N. Smirnov, V.P. Eismont, N.P. Filatov, J. Blomgren, H. Condé, A.V. Prokofiev, P.U. Renberg, and N. Olsson, Phys. Rev. C70 (2004) 054603.

[15] R. Nolte, M.S. Allie, P.J. Binns, F.D. Brooks, A. Buffler, V. Dangendorf, K. Langen, J.P. Meulders, W.D. Newhauser, F. Ross, and H. Schuhmacher, J. Nucl. Sci. Tech. Suppl. 2, vol. 1 (2002) 311. 
[16] O. Shcherbakov, A. Donets, A. Evdokimov, A. Fomichev, T. Fukahori, A. Hasegawa, A. Laptev, V. Maslov, G. Petrov, S. Soloviev, Y. Tuboltsev, and A. Vorobyev, J. Nucl. Sci. Tech. Suppl. 2, vol. 1 (2002) 230.

[17] P. Staples, P. W. Lisowski, and N. W. Hill, Bull. Am. Phys. Soc. 40 (1995) 962; P. Staples, private communication (1996). 